\title{
Regulation of myostatin expression is associated with growth and muscle development in commercial broiler and DMC muscle
}

\author{
Tengfei Dou ${ }^{1} \cdot{\text { Zhengtian } \mathrm{Li}^{1} \cdot \mathrm{Kun} \mathrm{Wang}^{1} \cdot \text { Lixian Liu }^{1} \cdot \text { Hua Rong }}^{1} \cdot$ Zhiqiang Xu$^{2} \cdot$ Ying Huang $^{1} \cdot$ Dahai Gu$^{1}$. \\ Xiaobo Chen ${ }^{1}$. Wenyuan $\mathrm{Hu}^{1}$. Jiarong Zhang ${ }^{1}$. Sumei Zhao ${ }^{1}$ - Markandeya Jois ${ }^{3}$. Qihua $\mathrm{Li}^{1}$. Changrong Ge ${ }^{1}$. \\ Marinus F. W. te Pas ${ }^{4,5,6}$. Junjing Jia ${ }^{1}$
}

Received: 3 April 2017 / Accepted: 26 April 2018 / Published online: 8 May 2018

(c) The Author(s) 2018

\begin{abstract}
Myostatin is a negative regulator of skeletal muscle growth. Muscle tissue is the largest tissue in the body and influences body growth. Commercial Avian broiler chickens are selected for high growth rate and muscularity. Daweishan mini chickens are a slow growing small-sized chicken breed. We investigated the relations between muscle (breast and leg) myostatin mRNA expression and body and muscle growth. Twenty chickens per breed were slaughtered at 0,30,60,90, 120, and 150 days of age. Body and muscle weights were higher at all times in Avian chickens. Breast muscle myostatin expression was higher in Avian chickens than in Daweishan mini chickens at day 30. Myostatin expression peaked at day 60 in Daweishan mini chickens and expression remained higher in breast muscle. Daweishan mini chickens myostatin expression correlated positively with carcass weight, breast and leg muscle weight from day 0 to 60 , and correlated negatively with body weight from day 90 to 150 , while myostatin expression in Avian chickens was negatively correlated with carcass and muscle weight from day 90 to 150 . The results suggest that myostatin expression is related to regulation of body growth and muscle development, with two different regulatory mechanisms that switch between days 30 and 60 .
\end{abstract}

Keywords Commercial broiler chicken $\cdot$ Daweishan mini chicken $\cdot$ Myostatin $\cdot$ mRNA expression $\cdot$ Muscle weight . Growth rate

Tengfei Dou, Zhengtian Li, Kun Wang and Lixian Liu have contributed equally to this work.

Electronic supplementary material The online version of this article (https://doi.org/10.1007/s11033-018-4187-7) contains supplementary material, which is available to authorized users.

Marinus F. W. te Pas

marinus.tepas@wur.nl

Changrong Ge

gcrzal@126.com

Junjing Jia

junjingli2013@qq.com

1 Yunnan Provincial Key Laboratory of Animal Nutrition and Feed, Yunnan Agricultural University, Kunming 650201, Yunnan Province, People's Republic of China

2 Faculty of Food Science, Yunnan Agricultural University, Kunming 650201, Yunnan Province, People's Republic of China

\section{Introduction}

Muscle tissue is the largest tissue in the body and may directly influence whole body growth. Myostatin regulates muscle fibre growth [1] and muscle development via regulating satellite cell activation and renewal [2]. In broiler chicken myostatin haplotypes were reported to be associated

3 Department of Agricultural Sciences, School of Life Sciences, Faculty of Science, Technology and Engineering, La Trobe University, Bundoora, VIC 3086, Australia

4 Animal Breeding and Genomics Centre, Wageningen UR Livestock Research, Wageningen University and Research Centre, Building 107, Radix, Droevendaalsesteeg 1, 6708 PB Wageningen, The Netherlands

5 Kunming Agricultural University, Kunming 650201, Yunnan Province, People's Republic of China

6 Dali University, Dali, People's Republic of China 
with body weight [3]. The relationship between myostatin mRNA expression, growth rate, muscle mass, and body weight in chickens is poorly understood. We used commercial Avian broiler (AB) and Daweishan mini chickens (DMC) as a model system to investigate these relationships. Broiler chickens are characterized by a high body weight at slaughter and extremely fast growth rate from hatching to slaughter [4, 5], while DMC are a slow growing low body weight breed [6]. The combination of these two breeds provides a perfect model system to investigate the biological mechanisms underlying growth rate and body weight in chickens. The objective of this research was to investigate the relationship between myostatin expression in breast and leg muscle and whole body growth rate, muscle development, and growth rate.

\section{Materials and methods}

\section{Animals and experiments}

This study was approved by the Animal care and use committee of the Yunnan Province of P. R. China and all the experiments complied with the requirements of the directory of the Ethical Treatment of Experimental Animals of China. DMC were purchased from the Chicken Farm of Yunnan Agricultural University within the first day of life. Commercial AB were purchased from the Chicken Farm (Kunming Zhengda Group, a source from the American IVY International Co., LTD). This study used 120 chickens per breed. At each time point $(0,30,60,90,120$, and 150 days of age) 20 animals per breed were sacrificed. The chicken were reared under standard conditions on starter diets to day 30 , and then on adult chicken diets to day 150 . Table 1 shows the diet compositions and some egg characteristics of the two chicken breeds.

The chickens had free access to feed and water during the entire rearing period. The chickens were reared in an environmentally controlled room. The brooding temperature was maintained at $35^{\circ} \mathrm{C}$ for the first 2 days, and then decreased gradually to $22{ }^{\circ} \mathrm{C}$ until 30 days. At 30 days of age, the chickens were randomly allocated to individual metabolism cages in an enclosed room, with ambient temperatures varying from 21 to $24{ }^{\circ} \mathrm{C}$ on a light:dark cycle of $12: 12 \mathrm{~h}$.

\section{Measurement of growth performance and carcass traits}

Chickens were weighed on a tarred digital scale (Shanghai Yizhan weighing apparatus limited company, YZ $0.01-10 \mathrm{~kg}$, China). Body weights were determined in the morning following a $16 \mathrm{~h}$ fasting. The body weights were determined until week 20 because the DMC reached sexual maturity at approximately 20 weeks. The carcass weight was measured after the blood and feathers had been removed. After removal of the oesophagus, trachea, gastrointestinal tract, pancreas, spleen, and gonads the semi-eviscerated weight was measured, and after removal of the head, heart, claws, liver, glandular stomach, gizzard, and abdominal fat the eviscerated weight was measured. The dressing percentage was calculated by dividing the carcass weight by the body weight. The percentage of carcass weight originating from of each carcass trait (eviscerated weight, semi-eviscerated weight, breast muscle weight, leg muscle weight, and abdominal fat weight) was calculated by dividing each trait by the carcass weight [7].
Table 1 Compositions and nutrient levels of the chicken diets, and egg characteristics of the two chicken breeds

\begin{tabular}{lll}
\hline & Avian broiler chicken & Daweishan mini chicken \\
\hline Age & 40 weeks-old & 30 weeks-old \\
Nutrition & & \\
Metabolizable energy $\left(\mathrm{Kcal} \mathrm{kg}^{-1}\right)$ & 2760 & 2750 \\
Crude protein $(\%)$ & 16.0 & 15.6 \\
Calcium (\%) & 3.07 & 3.00 \\
Total phosphorus $(\%)$ & 0.66 & 0.60 \\
Available phosphorus (\%) & 0.37 & 0.38 \\
Salt (\%) & 0.37 & 0.37 \\
Lys $(\%)$ & 0.82 & 0.76 \\
Met $(\%)$ & 0.42 & 0.35 \\
Methionine + cystinol (\%) & 0.70 & 0.63 \\
Egg weight (mean \pm SD) & $57.63 \pm 3.44$ & $35.52 \pm 4.10$ \\
Egg shape index (mean \pm SD) & $1.37 \pm 0.07$ & $1.30 \pm 0.04$ \\
Chick birth weight $(\mathrm{Mean} \pm \mathrm{SD})$ & $48.19 \pm 4.02$ & $20.70 \pm 2.50$ \\
\hline
\end{tabular}

Chickens received the starter diet from day 0 to 30 . From day 30 to 150 the chickens received the adult chicken diet 


\section{Myostatin real-time PCR}

Real-time PCR was performed to quantify skeletal muscle myostatin mRNA expression as described previously [8]. Breast and leg muscle samples were collected within 20 min after chickens were sacrificed. Samples were immediately placed in sterile tubes (RNase-free), snap frozen in liquid nitrogen, and stored at $-80{ }^{\circ} \mathrm{C}$. Total RNA was isolated using Trizol-Reagent (Invitrogen, USA) and reverse transcribed using oligo (dT) 12-18, random primers, and M-MLV reverse transcriptase (Invitrogen, USA).

Real-time PCR analysis was performed using the iCycler Real Time Detection System (Bio-Rad Laboratories, Inc. USA) and SYBR Green master mix (iQTM SYBRGreen ${ }^{\circledR}$ Supermix, Dalian TaKaRa Biotechnology Co. Ltd. Add). The myostatin primers used were 5'-GCTTTT GATGAGACTGGACGAG-3' (forward) and 5'-AGCGGG TAGCGACAACATC-3' (reverse), and the annealing temperature was $60{ }^{\circ} \mathrm{C}$. The $18 \mathrm{~S}$ rRNA gene was used as a reference: 5'CGCGTGCATTTATCAGACCA-3' (forward) and 5'-ACCCGTGGTCACCATGGTA-3' (reverse), annealing temperature $58{ }^{\circ} \mathrm{C}$. Primers were commercially synthesized (Shanghai Shenggong Biochemistry Company P.R.C). The PCR reactions were performed in $25 \mu \mathrm{l}$ volumes containing $12.5 \mu \mathrm{l}$ of $\mathrm{iQ}^{\mathrm{TM}}$ SYBR Green Supermix, $0.5 \mu \mathrm{l}\left(10 \mathrm{mmol}^{-1}\right)$ of each primer, and $1 \mu \mathrm{l}$ of cDNA. Amplification and detection of products was performed with the following cycle profile: one cycle of $95{ }^{\circ} \mathrm{C}$ for $2 \mathrm{~min}$, and 40 cycles of $95^{\circ} \mathrm{C}$ for $15 \mathrm{~s}$, annealing temperature for $30 \mathrm{~s}$, and $72{ }^{\circ} \mathrm{C}$ for $30 \mathrm{~s}$, followed by a final cycle of $72{ }^{\circ} \mathrm{C}$ for $10 \mathrm{~min}$. The specificity of the amplification product was verified by electrophoresis on a $0.8 \%$ agarose gel and DNA sequencing.

\section{Statistical analysis}

Carcass trait data were expressed as the mean $\pm \mathrm{SE}$ for the two breeds. SAS version 9.3 was used for all analyses as described by Arounleut et al. [9]. Myostatin mRNA expression levels were expressed relative to $18 \mathrm{~S}$. Differences in temporal gene expression and comparisons between breast and leg muscle were analyzed using a $T$ test. Both breeds were tested individually across all time points (0-150 day of age). Correlation analyses between myostatin expression and carcass traits were performed using the Spearman correlation procedure, which was chosen because of the nonparametric nature of the data. The CORR procedure of SAS (SAS version 9.3) was used to perform the correlations. Significance and residual values were calculated using a two-way ANOVA. $P<0.05$ was used to determine statistical significance.

\section{Results}

\section{The model system}

Figure 1 shows the growth rate (Fig. 1a) and body weight (Fig. 1b) data for the two chickens. At all ages the $\mathrm{AB}$ line chickens showed a higher growth rate than the DMC chickens. The commercial AB line chickens showed a high increased daily growth rate from hatching to 5 weeks of age after which the growth rate decreased. The DMC chickens showed a small increase in average daily growth rate until week 8 followed by a small decline of the average daily growth. The DMC chickens showed no peak like the $\mathrm{AB}$ line chickens for growth rate. As a consequence of this the body weight of the $\mathrm{AB}$ line chickens increased more sharply than the DMC and reached a much higher body weight at 20 weeks (Fig. 1b).
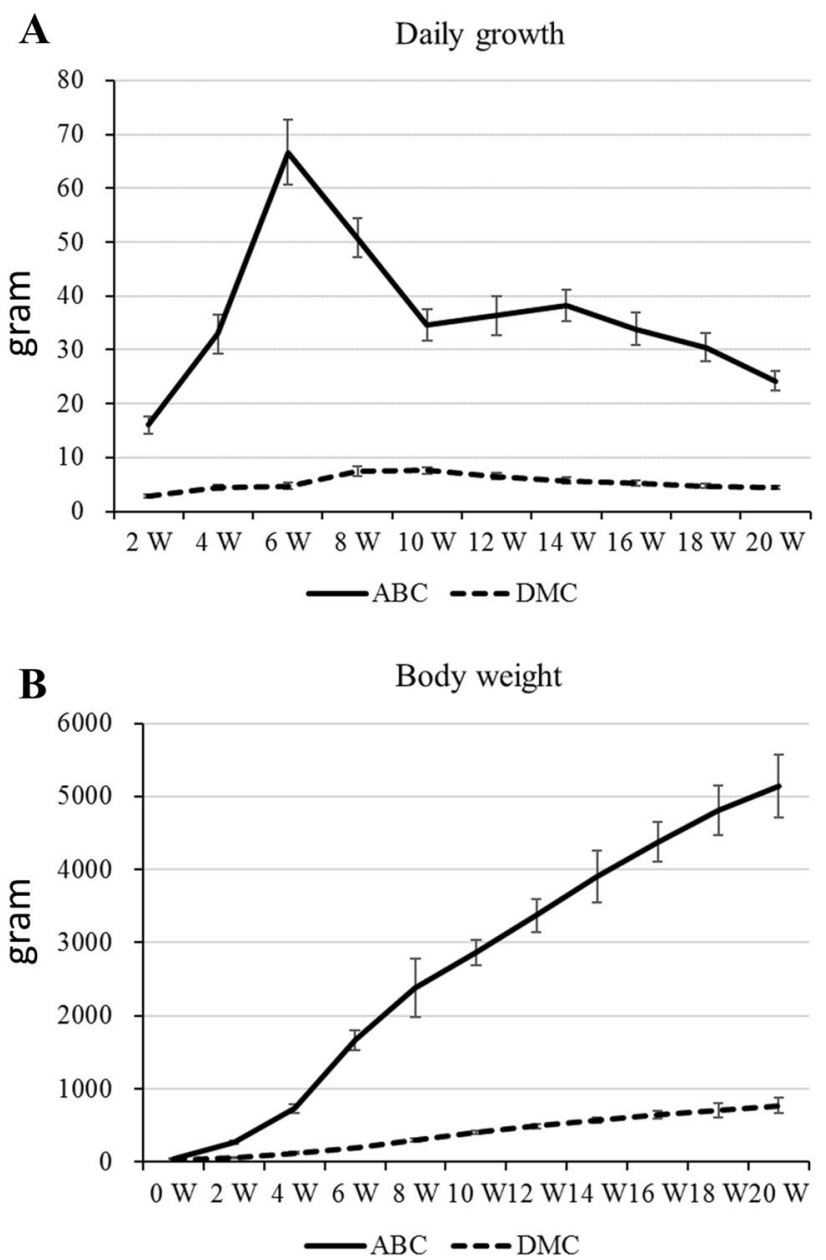

Fig. 1 Daily growth rate (a) and body weight (b) of the ABC and the DMC. Data are in grams 
Figure 2 shows the carcass weight gain and muscle development. The carcass weights (Fig. 2a) of the DMC were significantly lower than the commercial $\mathrm{AB}$ during the entire growth period. The carcass weight, breast muscle weight, and leg muscle weight (Fig. 2a, c, e) increased faster after day 30 than during the first 30 days of life. The dressing percentage (Fig. 2b), which is the amount of meat on the carcass, indicates that muscle content was higher in the $\mathrm{AB}$ carcasses. Both breast (Fig. 2c) and leg muscle (Fig. 2e) growth rates were closely related to carcass weight. Higher breast muscle percentage (Fig. 2d), but not leg muscle percentage (Fig. 2f) was observed in the AB compared to the DMC.
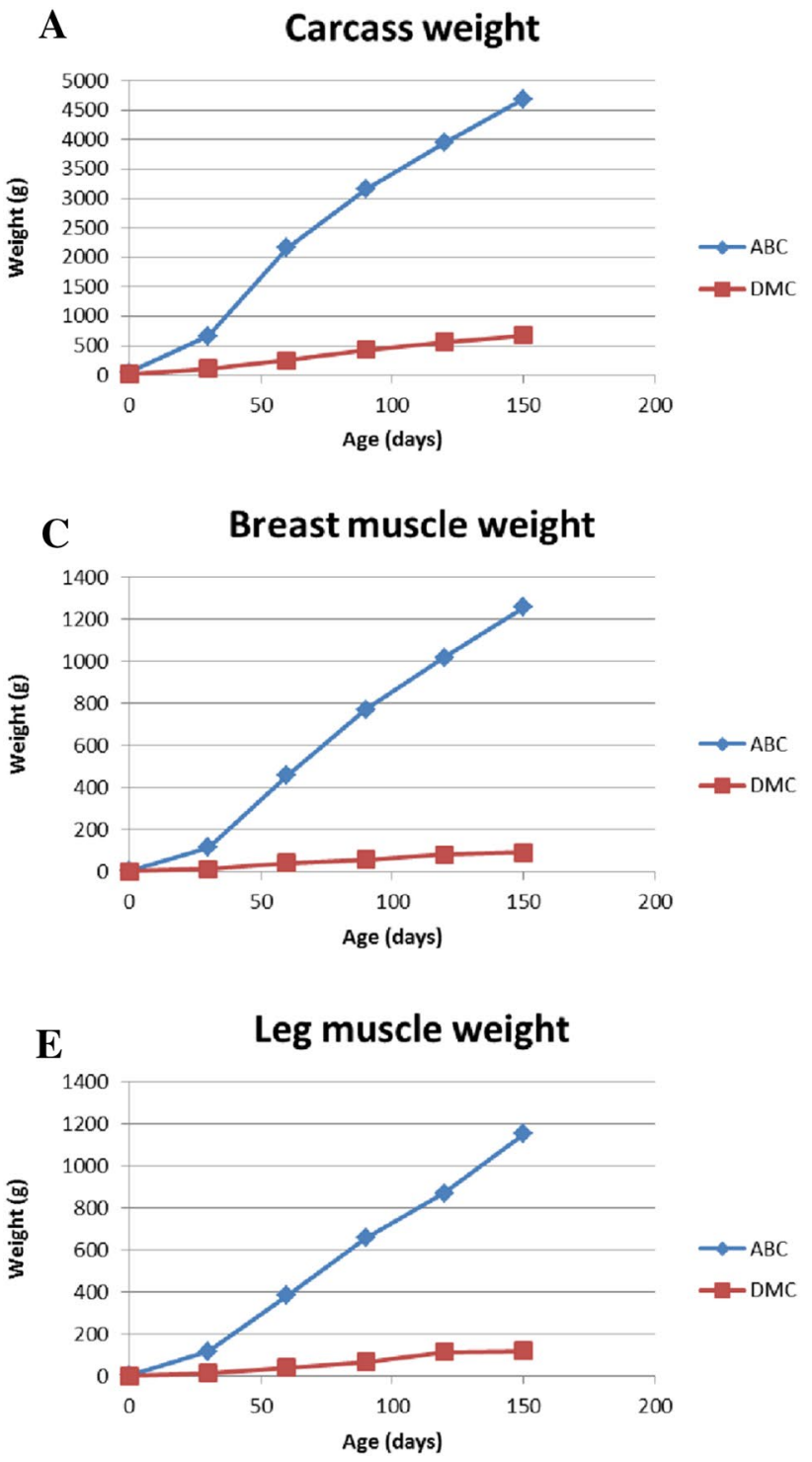

Fig. 2 Developmental changes in commercial $\mathrm{AB}(\mathrm{ABC})$ and $\mathrm{DMC}$ (DMC) from birth to 150 days of age. a, c, e Measured weights (g) of carcass, breast muscle, and leg muscle, respectively; b, d, f calculated percentages of dressing, breast muscle, and leg muscle, respectively. The dressing percentage was the carcass weight divided by the body
These observations were confirmed by additional carcass measurements (Table 2, Supplementary material 1).

\section{Myostatin mRNA expression}

Figure 3 shows myostatin expression levels of breast and leg muscle. Myostatin expression in leg muscle was higher than in breast muscle in the $\mathrm{AB}$, with the largest difference observed on days 60-120. The smallest difference was observed on days 0 and 30. Differences between breast and leg muscle myostatin expression in the DMC were only observed at day 60 .

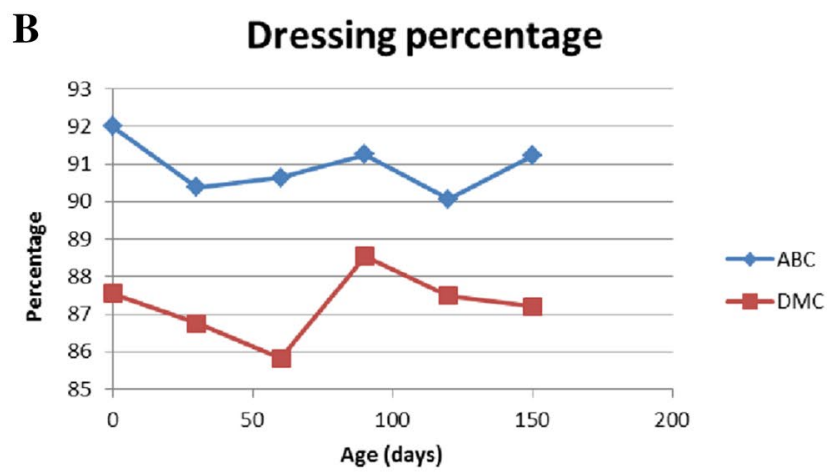

\section{Breast muscle percentage}
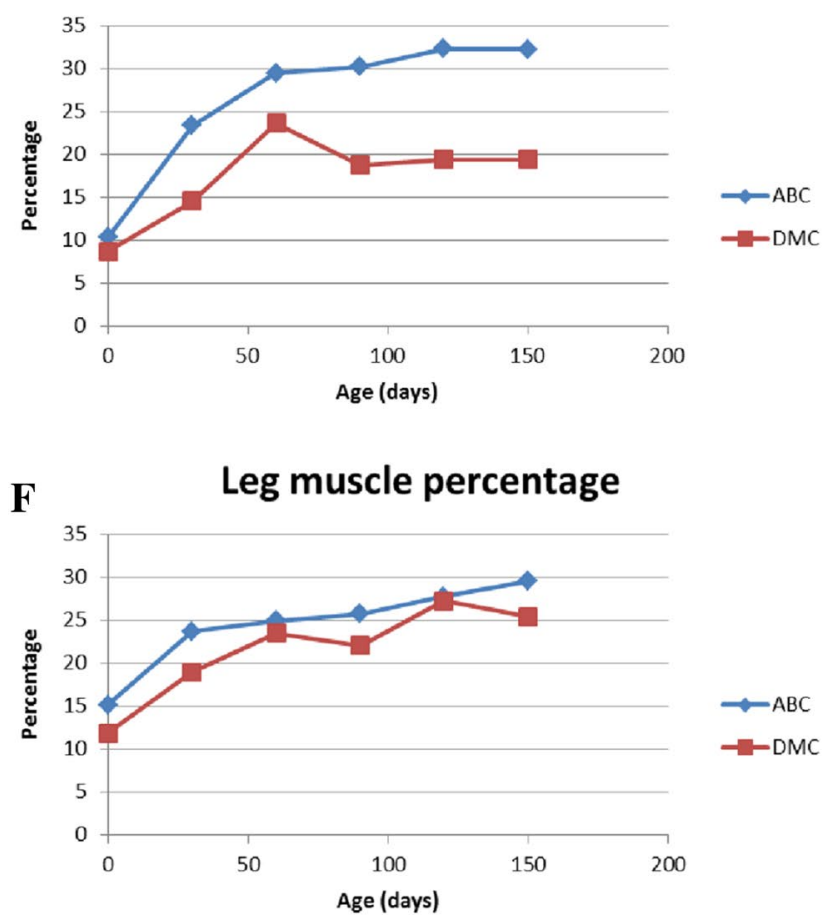

weight. The muscle percentages were calculated by dividing muscle weight by carcass weight. All measurements differed between ABC and DMC at all time points except for breast muscle percentage (d) at birth (0 days) and leg muscle percentage at day 0,30, 60, and 120 
Table 2 Carcass measurements of the Avian broiler line and the DMC breed at all sampled ages

\begin{tabular}{|c|c|c|c|c|c|c|}
\hline \multirow[t]{2}{*}{ Carcass traits } & \multicolumn{6}{|l|}{ Age } \\
\hline & 0 days & 30 days & 60 days & 90 days & 120 days & 150 days \\
\hline \multicolumn{7}{|l|}{$\mathrm{AB}$} \\
\hline Carcass weight $(\mathrm{g})^{\mathrm{a}}$ & 44.38 & 662.85 & 2156.57 & 3158.65 & 3941.50 & 4690.67 \\
\hline Semi-eviscerated weight $(\mathrm{g})^{\mathrm{b}}$ & 39.81 & 606.35 & 1762.16 & 2839.19 & 3557.87 & 4283.46 \\
\hline Eviscerated weight $(\mathrm{g})^{\mathrm{c}}$ & 34.04 & 494.04 & 1541.42 & 2549.19 & 3129.87 & 3884.63 \\
\hline Breast muscle weight $(\mathrm{g})$ & 3.56 & 116.08 & 457.55 & 770.17 & 1017.50 & 1257.67 \\
\hline Leg muscle weight (g) & 5.14 & 117.54 & 384.47 & 657.23 & 869.67 & 1151.62 \\
\hline Dressing percentage $(\%)^{\mathrm{d}}$ & 92.01 & 90.38 & 90.63 & 91.25 & 90.07 & 91.22 \\
\hline Semi-eviscerated percentage $(\%)^{\mathrm{e}}$ & 82.50 & 82.65 & 81.72 & 82.14 & 81.42 & 83.31 \\
\hline Eviscerated percentage $(\%)^{\mathrm{e}}$ & 70.53 & 74.49 & 71.52 & 73.77 & 71.55 & 75.58 \\
\hline Breast muscle percentage $(\%)^{\mathrm{e}}$ & 10.41 & 23.39 & 29.54 & 30.23 & 32.38 & 32.29 \\
\hline Leg muscle percentage $(\%)^{\mathrm{e}}$ & 15.18 & 23.71 & 24.99 & 25.79 & 27.80 & 29.58 \\
\hline Abdominal fat percentage $(\%)^{\mathrm{e}}$ & & & & 4.07 & 5.56 & 5.33 \\
\hline Abdominal fat weight $(\mathrm{g})^{\mathrm{e}}$ & & & & 101.24 & 167.33 & 196.00 \\
\hline \multicolumn{7}{|l|}{ DMC } \\
\hline Carcass weight $(\mathrm{g})^{\mathrm{a}}$ & 18.12 & 107.23 & 251.3 & 434.29 & 564.28 & 673.23 \\
\hline Semi-eviscerated weight $(\mathrm{g})^{\mathrm{b}}$ & 15.6 & 88.84 & 214.5 & 365.51 & 502.28 & 577.31 \\
\hline Eviscerated weight $(\mathrm{g})^{\mathrm{c}}$ & 12.96 & 79.19 & 180.83 & 306.1 & 415.86 & 477.59 \\
\hline Breast muscle weight $(\mathrm{g})$ & 1.13 & 11.55 & 41.16 & 57.77 & 80.9 & 92.53 \\
\hline Leg muscle weight (g) & 1.52 & 15.05 & 42.43 & 67.83 & 114.14 & 121.46 \\
\hline Dressing percentage $(\%)^{\mathrm{d}}$ & 87.55 & 86.76 & 85.83 & 88.54 & 87.49 & 87.2 \\
\hline Semi-eviscerated percentage $(\%)^{\mathrm{e}}$ & 75.14 & 71.89 & 73.23 & 74.67 & 77.88 & 74.84 \\
\hline Eviscerated percentage $(\%)^{\mathrm{e}}$ & 62.45 & 64.09 & 61.86 & 62.58 & 64.41 & 61.81 \\
\hline Breast muscle percentage $(\%)^{\mathrm{e}}$ & 8.71 & 14.62 & 23.67 & 18.8 & 19.43 & 19.45 \\
\hline Leg muscle percentage $(\%)^{\mathrm{e}}$ & 11.88 & 18.98 & 23.55 & 22.12 & 27.28 & 25.47 \\
\hline Abdominal fat percentage $(\%)^{\mathrm{e}}$ & & & & 3.14 & 5.08 & 4.51 \\
\hline Abdominal fat weight $(\mathrm{g})^{\mathrm{e}}$ & & & & 9.52 & 20.43 & 20.31 \\
\hline
\end{tabular}

Carcass measurements were done after subsequently dissection of various organs as detailed in the footnotes

${ }^{a}$ The carcass weight was measured after the blood and feathers had been dissected

${ }^{\mathrm{b}}$ The semi-eviscerated weight was measured after subsequent dissection of the oesophagus, trachea, gastrointestinal tract, pancreas, spleen, and gonads

${ }^{c}$ the eviscerated weight was measured after subsequent dissection of the head, heart, claws, liver, glandular stomach, gizzard and abdominal fat

${ }^{\mathrm{d}}$ The dressing percentage was determined as the proportion of the carcass weight of the body weight

${ }^{\mathrm{e}}$ The proportions of the weights from each of the carcass traits (eviscerated weight, semi-eviscerated weight, breast muscle weight, leg muscle weight, and abdominal fat weight) of the carcass weight were calculated as eviscerated percentage, semi-eviscerated percentage, breast muscle percentage, leg muscle percentage, and abdominal fat percentage, respectively
Breast muscle myostatin expression was higher in the $\mathrm{AB}$ than the DMC at day 30. In contrast, breast and leg muscle myostatin expression was higher in DMC than $\mathrm{AB}$ from day 60 to day 150. Myostatin expression in DMC reached a peak level at day 60 in both muscles, which was not observed in the AB. Although breast muscle myostatin expression decreased after day 60 in the $\mathrm{DMC}$, it remained relatively high compared to $\mathrm{AB}$. Leg muscle myostatin expression also decreased after day 60 in the DMC, reaching the same overall levels observed in the $\mathrm{AB}$ on days 90 and 120 . There remained a small but significant difference in myostatin expression between the two breeds at days 120 and 150 .

\section{Correlation between animal traits and myostatin expression}

Figure 3 shows differences in myostatin expression between day 0 and day 30 , and from day 60 onwards. This indicates that important changes in the regulation and effect of myostatin may occur between 30 and 60 days of age. Therefore, we correlated myostatin expression with the measured production 
Fig. 3 Myostatin expression of the breast muscle of DMC (BM$\mathrm{DMC})$ and commercial $\mathrm{AB}$ (BM-ABC), and the leg muscle of DMC (LM-DMC) and commercial $\mathrm{AB}$ (LM-ABC) at 0 , $30,60,90,120$, and 150 days of age. Data are expressed as the mean ratio $\pm \mathrm{SE}$ of specific myostatin mRNA:18S rRNA for each time point from the two breeds. Myostatin expression differences $(\mathrm{P}<0.05)$ were evaluated between time points within each breed (lower case letters) and between the two breeds for each time point $(*)$
A

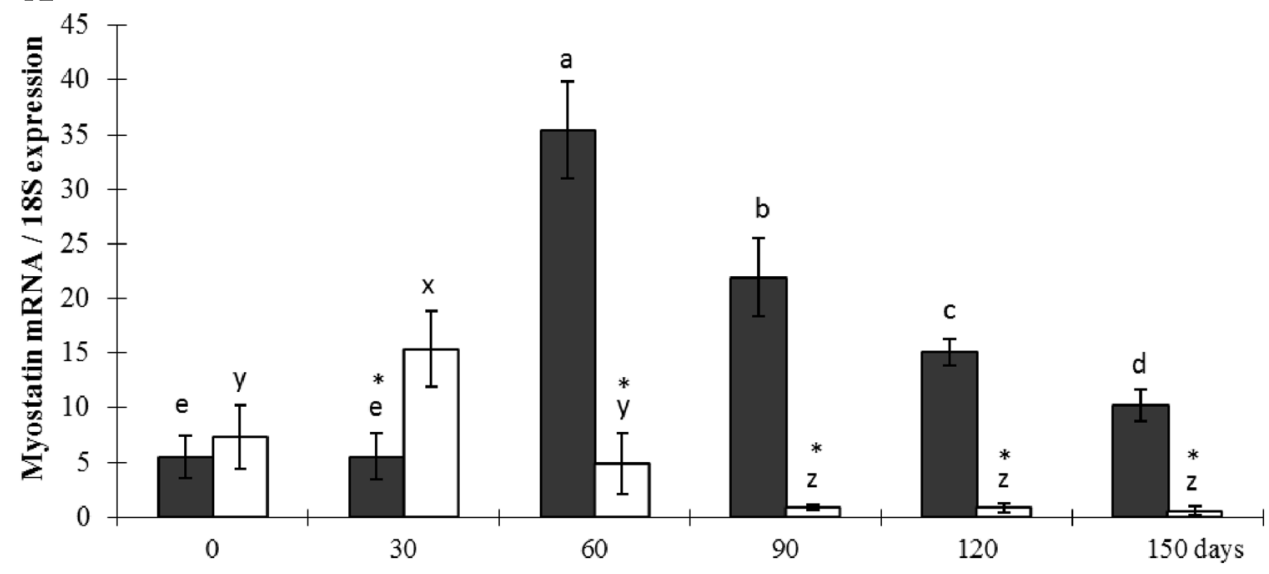

B

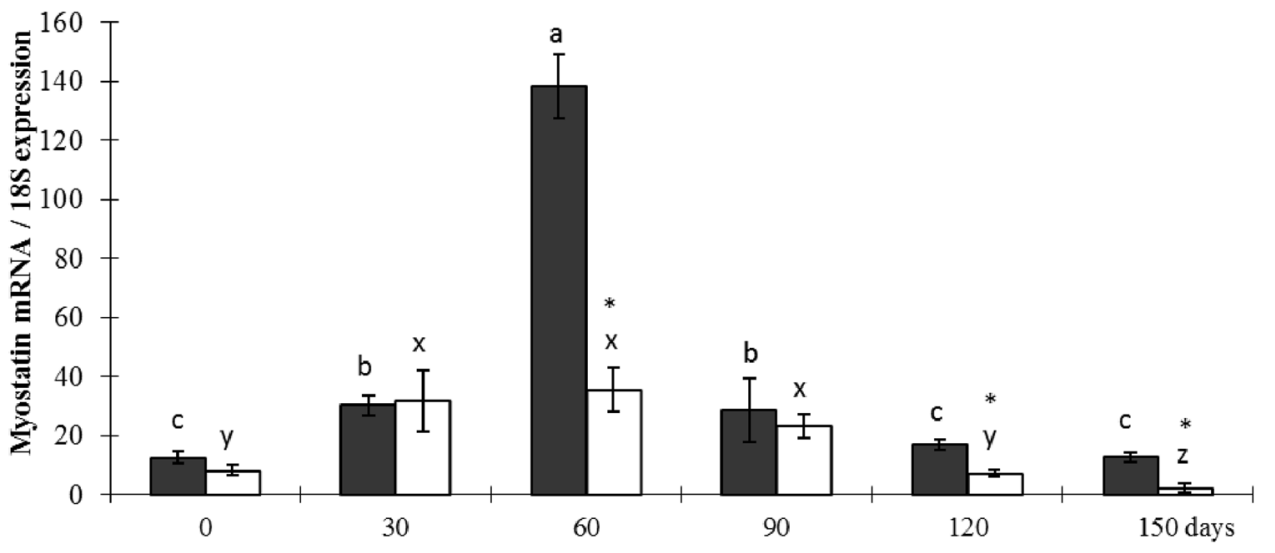

traits for time points ranging from day 0 to 30 , day 60 to 150 (Table 3), day 0 to 60 , and day 90 to 150 (Table 4). The results differ for days 30 to 60 as the end of period 1, the period where the largest differences in myostatin expression were found (Fig. 3). The analyses showed high similarity between the breeds for the correlations. The correlations for the commercial AB were significant for day $0-30$ and day $60-150$, but not day 0-60 and day 90-150. A reverse in the direction of the correlation was observed for the DMC. This may indicate that myostatin expression is differently regulated between the two chicken breeds.

Additional correlations between the muscle/meat characteristics were also calculated (Table 5, day 0-60 and day 90-150). The correlations were as expected for carcass quality data and did not differ between the two analyses differing for either day 60 or 90 separating the two developmental periods; high correlations were found between body weight and carcass traits measured after slaughter.

\section{Discussion}

Commercial broiler chickens have been subjected to strong human-driven selection leading to remarkable phenotypic changes in morphology, such as increased muscle growth and physiology leading to a more than $300 \%$ increase in body growth rates [10-12], accompanied by significant increases in metabolic rates [7, 13] and higher incidence of sudden death syndrome [14], associated with smaller organs. The internal organs of commercial AB develop slower, remain smaller in size, and have limited oxygen supply compared to the Daweishan chickens [15]. We observed a high correlation between body weight and carcass traits, indicating balanced growth in both breeds. The lower correlation comparing $\mathrm{AB}$ and DMC for the eviscerated weight from day 90 to 150 revealed higher internal organ content in the DMC. 


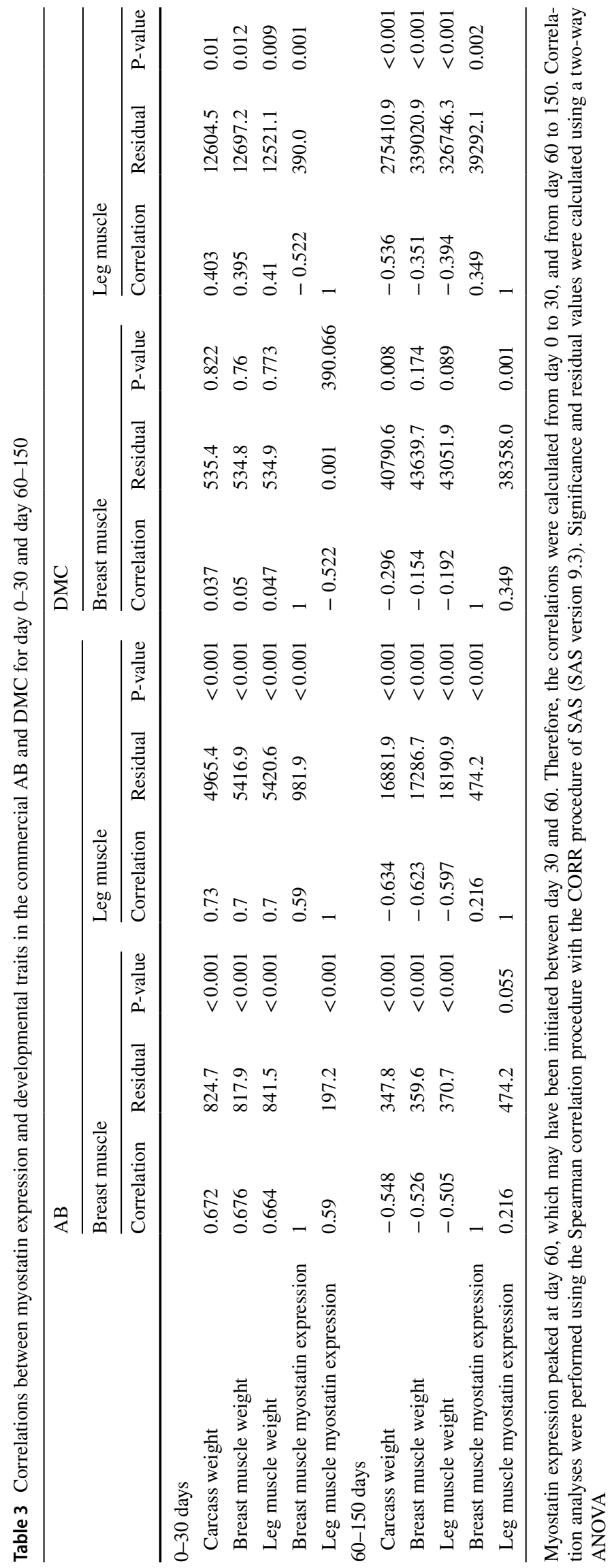




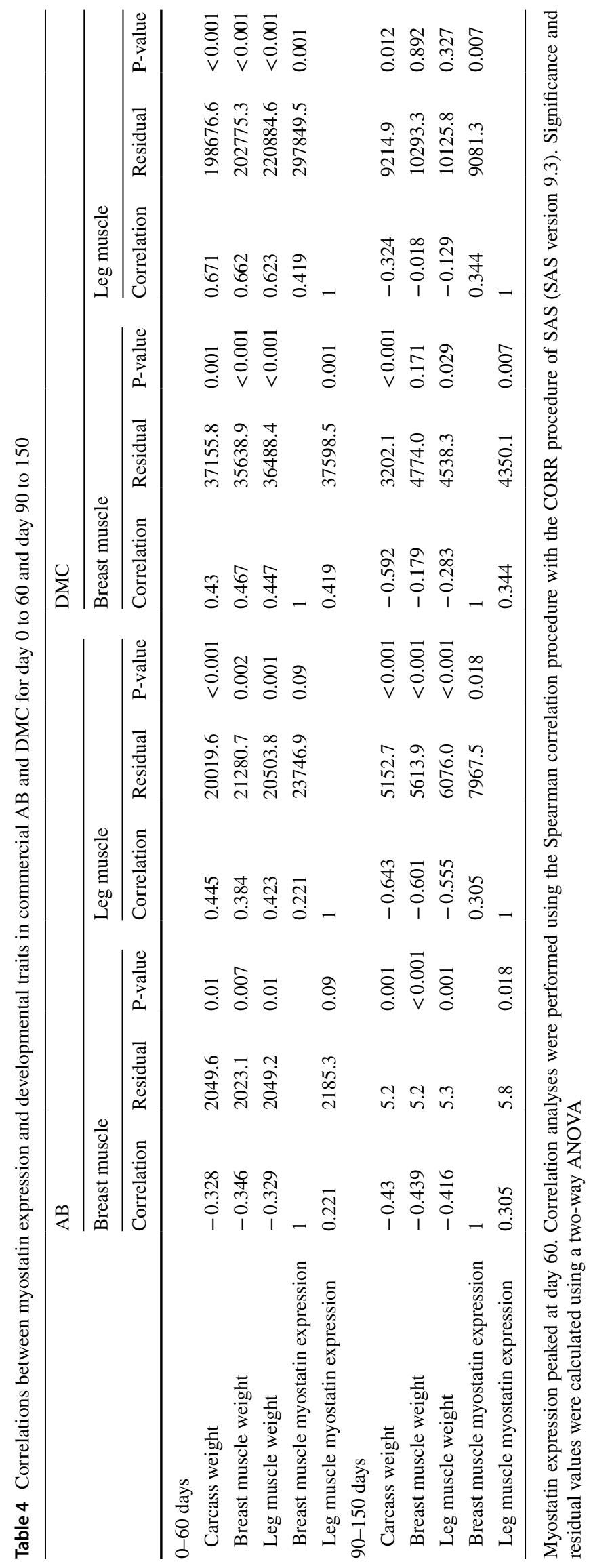




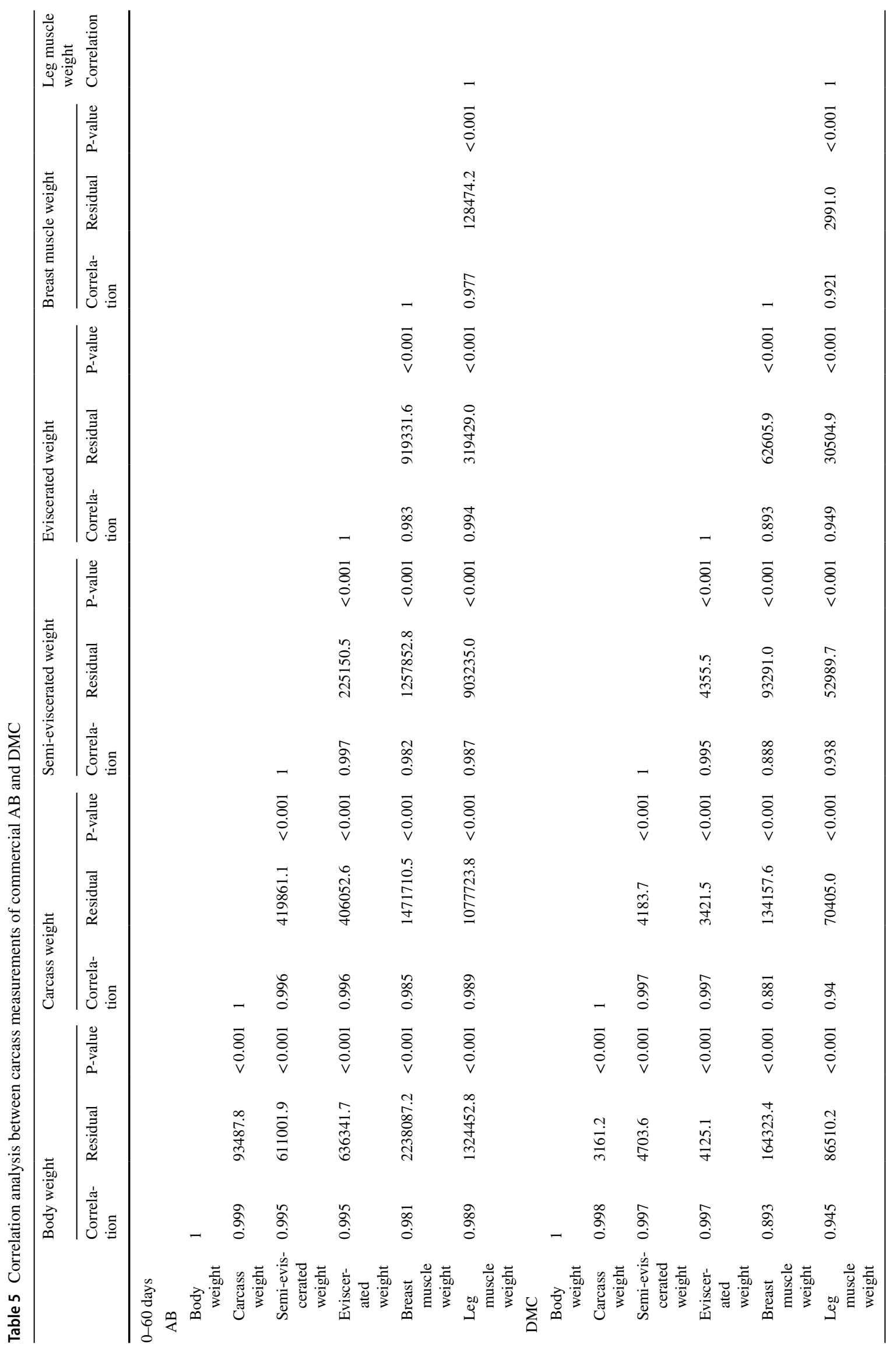




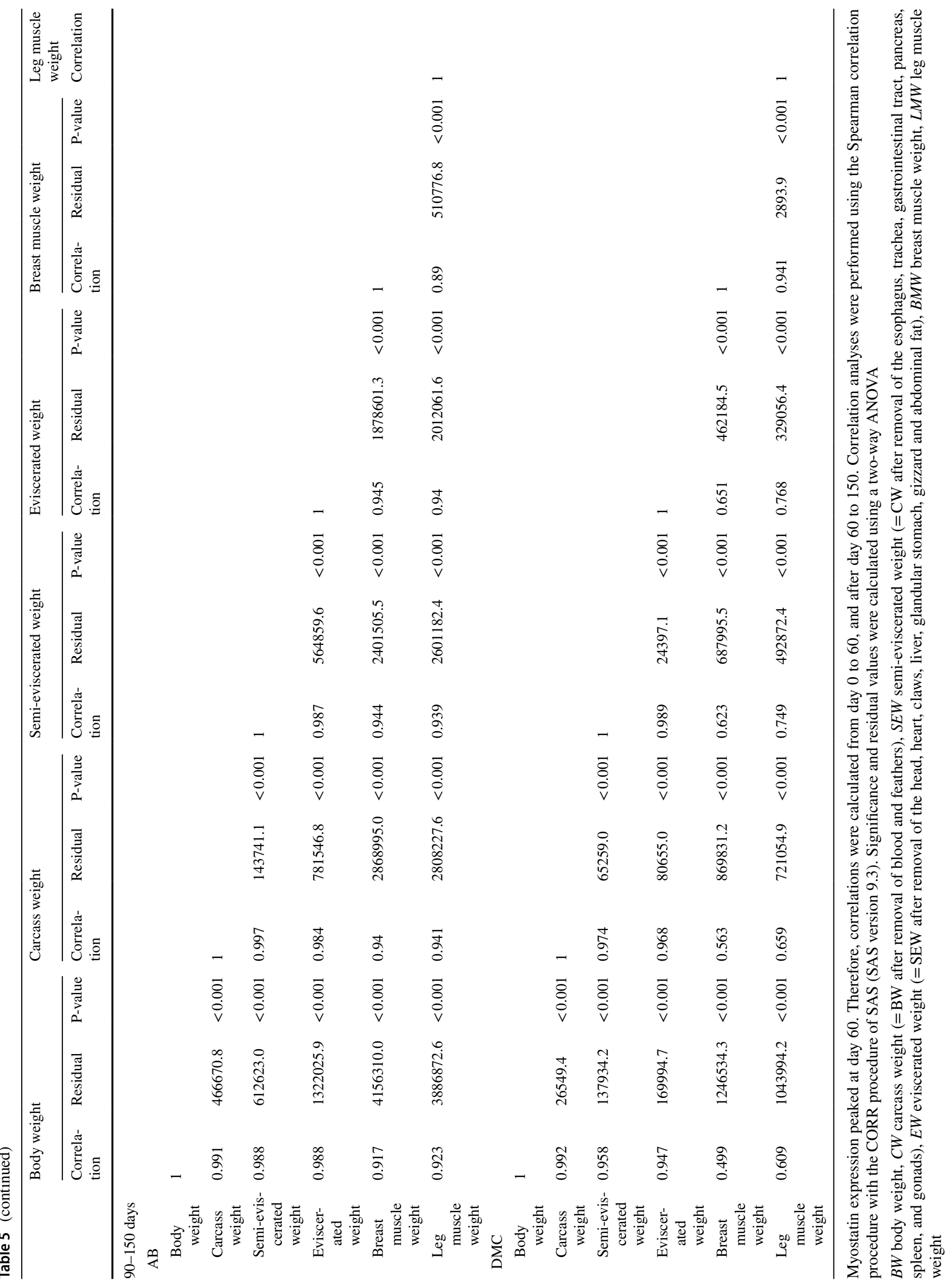


DMC displayed low body and muscle growth rates, leading to a lower body weight compared to the commercial $\mathrm{AB}$. Identifying the genetic changes underlying these developmental differences would provide new insight into the biological mechanisms by which genetic variation shapes phenotypic diversity [16]. Our results provide evidence for major differences in body growth rates and muscular development between the two breeds. The commercial AB are characterized by an extremely high dressing percentage $(>90 \%)$ due to high muscularity $(>60 \%$ for breast and leg muscles only), both of which were more moderate in the DMC (muscle content $45 \%$ for these two muscles). We conclude that comparing these two breeds together provides a large phenotypic contrast, providing an excellent model system to investigate the molecular biological mechanisms underlying the physiology of selection pressures applied to commercial AB. The lower dressing percentage of DMC compared to commercial $\mathrm{AB}$ observed here indicates that DMC have relatively higher internal organ weights. These differences may indicate breeding effects due to selection for high growth rate and muscle content in the commercial AB.

Breast and leg muscle myostatin expression in DMC peaked at day 60 . This suggests that this developmental time point represents a crucial regulatory stage. Breast muscle myostatin expression at day 30 is higher in commercial $\mathrm{AB}$ than DMC, suggesting reduced muscle development in the commercial AB. Indeed a delay in the development of both muscles — especially breast muscle—and body growth rates was associated with increased myostatin expression at day 30 in the commercial AB. Nevertheless, the growth rate of commercial $\mathrm{AB}$ is already much higher than DMC, which may be related to the selection background of the breed.

From day 60 onwards, breast and leg muscle myostatin expression was higher in the DMC than the commercial AB. Myostatin reduces muscle growth [1] and body/carcass growth rates [17], and the body/carcass growth and muscle development rates of commercial $\mathrm{AB}$ is much higher than DMC. This suggests that from day 60 onwards, myostatin is a regulator of both muscle and whole body growth.

The higher myostatin expression observed in the leg muscle compared to breast muscle suggests leg muscle growth rates are affected more by myostatin than breast muscle growth rates. Therefore, we hypothesize that the regulation of myostatin expression is part of the biological mechanism underlying the response to selection for increased breast muscle development and body growth rates in commercial AB. This is in agreement with Guernec et al. [18].

Our correlation analyses between myostatin expression and body and muscle growth suggest an important switch in regulatory mechanisms between 30 and 60 days of age. The observed correlations were higher in the time period ranging between day 0 to 30 and day 0 to 60 for the commercial $\mathrm{AB}$, and to a lesser extent in the time period ranging from day 60 to 150 than day 90 to 150 . The opposite was observed for the DMC. We concluded that (1) the switch in regulation of myostatin expression occurs between 30 and 60 days of age in both breeds, and (2) the myostatin effect is higher in the young commercial $\mathrm{AB}$ than the young DMC, while the reverse conclusion can be reached for the older chickens. Myostatin expression is highly regulated via different mechanisms. These mechanisms may induce the regulatory switch. The selection pressure in the commercial AB line may have affected these regulatory mechanisms, and as a consequence the mechanisms differ between the two chicken breeds. An alternative explanation of the observed myostatin effects may be that the regulation of muscle weights and body weights differs in the two breeds. In the time period ranging from day 90 to 150 , myostatin expression correlated negatively with body growth and leg muscle development in the commercial $\mathrm{AB}$, and with body growth in the DMC. Higher myostatin expression was associated with reduced body growth rates and muscle development. This suggests a direct biological effect of myostatin expression on these traits. The observed lower myostatin expression, especially in the breast muscle of the commercial $\mathrm{AB}$ compared to the DMC could result from selection for increased breast muscle weight and whole body growth rates. Because myostatin reduces the development and size of muscle tissue, lower myostatin expression could explain the biological regulatory mechanism by which through which selection results in increased body growth rate and muscle size.

While correlation was observed between myostatin expression and body and muscle growth in young animals (0-60 days), there was a lack of association between the myostatin expression between leg and breast muscle. This again indicates that myostatin expression is differently regulated between leg and breast muscle. This may indicate that the switch in regulation of myostatin expression between 30 and 60 days of age in both breeds has finished and therefore regulatory mechanisms differ.

The situation in young animals (0-60 days) is different. No correlation between myostatin expression and body and muscle growth was observed in the young $\mathrm{AB}$, suggesting myostatin expression is not involved in regulating growth rates in the high growth and muscularity broiler breed during early development.

In conclusion, our data suggest that regulation of myostatin expression may be part of a biological mechanism underlying selection for high growth rate, high muscularity, and high body weight in commercial AB. The biological effects differ before and after 60 days of age, at which point myostatin expression peaks in the slow growing chicken line. Furthermore, the biological mechanisms underlying the response to selection in breast and leg muscle seem to be different in the two growth periods. This suggests that 
regulation of myostatin represents only a part of the biological mechanisms at play.

Acknowledgements The Authors acknowledge Dr. Kyle Schachtschneider (Department of Radiology, University of Illinois, Chicago, USA) for English editing of the manuscript. This work was partially funded by National Natural Science Foundation of China (U1702232, 31560618, 31572359) (JJ and CG), Yunnan Provincial Natural Science Foundation (2016FA046, 2017IC048) (JJ), and National Science and Technology Program in the 13th Five-Year Plan (2015BAD03B03-4) (CG). The funders had no role in the study design, data collection and analysis, decision to publish, or preparation of the manuscript. The authors declare no competing financial interest.

Authors contribution Conceived and designed the experiments: JJ, SZ, DG, CG, MJ; Performed the experiments: TD, LL, HR, ZX, YH, XC, WH; Analyzed the data: TD, MtP; Contributed reagents/materials/ analysis tools: TD, LL, HR, WH, JZ, QL; Wrote the paper: TD, MtP; Organized funding: JJ, SZ, DG, CG; Completed calculations and participated in the interpretation of the results: TD, MtP.

\section{Compliance with ethical standards}

Conflict of interest The authors declare that they have no conflict of interest. The funding agencies had no influence on the experimental design and the conclusions.

Ethical approval All animal handling complied with the requirements of the directory of the Ethical Treatment of Experimental Animals of China.

Open Access This article is distributed under the terms of the Creative Commons Attribution 4.0 International License (http://creativeco mmons.org/licenses/by/4.0/), which permits unrestricted use, distribution, and reproduction in any medium, provided you give appropriate credit to the original author(s) and the source, provide a link to the Creative Commons license, and indicate if changes were made.

\section{References}

1. McPherron AC, Lawler AM, Lee SJ (1997) Regulation of skeletal muscle mass in mice by a new TGF- $\beta$ superfamily member. Nature 387:83-90

2. McCroskery S, Thomas M, Maxwell L, Sharma M, Kambadur M (2003) Myostatin negatively regulates satellite cell activation and self-renewal. J Cell Biol 162:1135-1147

3. Bhattacharya TK, Chatterjee RN (2013) Polymorphism of the myostatin gene and its association with growth traits in chicken. Poult Sci 92:910-915

4. Pym RAE, Farrell DJ (1977) A comparison of the energy and nitrogen metabolism of broilers selected for increased growth rate, food consumption and conversion of food to gain. Br Poult Sci 18:411-426

5. Marks HL (1979) Growth rate and feed intake of selected and nonselected broilers. Growth 43:80-90

6. Rong H, Chen XB, Xiong BL, Wan QS, Cao ZH, Chen BD, Wen S, Zhang X, Ge C, LI Q, Jia J (2011) Study on the germplasm characteristic of Yunnan DMC. J Yunnan Agric Univ 26:48-53

7. Scheele CW, Decuypere E, Vereijken PFG, Schreurs FJG (1992) Ascites in broilers. 2. Disturbances in the hormonal regulation of metabolic rate and fat metabolism. Poult Sci 71:1971-1984

8. Li QH, Xu ZQ, Liu L, Yu HX, Tao LL, Zhang X, Chen X, Gu D, Fan Y, Li X, Ge C, Tian Y, Jia J (2013) Effects of breeds and dietary protein levels on the growth performance, energy expenditure and expression of avUCP mRNA in chickens. Mol Biol Rep 40:2769-2779

9. Arounleut P, Bialek P, Liang LF, Upadhyay S, Fulzele S, Johnson M. Elsalanty M, Isales CM, Hamrick MW (2013) A myostatin inhibitor (propeptide-Fc) increases muscle mass and muscle fiber size in aged mice but does not increase bone density or bone strength. Exp Gerontol 48:898-904

10. Le Bihan-Duval E, Mignon-Grasteau S, Millet N, Beaumont C (1998) Genetic analysis of a selection experiment on increased body weight and breast muscle weight as well as on limited abdominal fat weight. Br Poult Sci 39:346-353

11. Havenstein GB, Ferket PR, Qureshi MA (2003) Growth, livability, and feed conversion of 1957 versus 2001 broilers when fed representative 1957 and 2001 broiler diets. Poult Sci 82:1500-1508

12. Havenstein GB, Ferket PR, Qureshi MA (2003) Carcass composition and yield of 1957 versus 2001 broilers when fed representative 1957 and 2001 broiler diets. Poult Sci 82:1509-1518

13. Buyse J, Michels H, Vloeberghs J, Saevels P, Aerts JM, Ducro B, Berckmans D, Decuypere E (1998) Energy and protein metabolism between 3 and 6 weeks of age of male broiler chickens selected for growth rate or for improved food efficiency. Br Poult Sci 39:264-272

14. Olkowski AA, Classen HL (1995) Sudden death syndrome in broiler chickens: a review. Poult Avian Biol Rev 6:95-105

15. Bowes VA, Julian RJ (1988) Organ weights of normal broiler chickens and those dying of sudden death syndrome. Can Vet J 29:153-156

16. Rubin CJ, Zody MC, Eriksson J, Meadows JR, Sherwood E, Webster MT, Jiang L, Ingman M, Sharpe T, Ka S, Hallböök F, Besnier F, Carlborg O, Bed'hom B, Tixier-Boichard M, Jensen P, Siegel P, Lindblad-Toh K, Andersson L (2010) Whole-genome resequencing reveals loci under selection during chicken domestication. Nature 464:587-591

17. Boman IA, Klemetsdal G, Nafstad O, Blichfeldt T, Våge DI (2010) Impact of two myostatin (MSTN) mutations on weight gain and lamb carcass classification in Norwegian White Sheep (Ovis aries). Genet Sel Evol 42:4. https://doi. org/10.1186/1297-9686-42-4

18. Guernec A, Berri C, Chevalier B, Wacrenier-Cere N, Le BihanDuval E, Duclos MJ (2003) Muscle development, insulin-like growth factor-I and myostatin mRNA levels in chickens selected for increased breast muscle yield. Growth Horm IGF Res 13:8-18 